\title{
Compositional Nature of Language to Represent Bimodal Visual- Audial Percepts
}

\author{
Elakya R, Himanshu Sinha, Prince Kumar, Singh Anubhav Gajendra, Shubham Gupta
}

\begin{abstract}
We describe the perceptual domain which have a composition domain and also which is rarely ever captured in the existed system. This has happened because they started to learn the composition structure directly. Compositional structures can be divided into separate domains. Keeping that in mind, we propose another way to deal with demonstrating bimodal perceptual areas that expressly relates unmistakable projections over every methodology and after that mutually learns a bimodal meager portrayal. Presently this model will empower compositionality crosswise over particular projections and sum up to percept's traversed by this compositional premise. For instance, our model can be prepared on red triangles and blue squares; yet, certainly will likewise have learned red squares and blue triangles. To test our model, we have procured another bimodal dataset including pictures and spoken articulations of hued shapes (hinders) in the table top setting.
\end{abstract}

Index Terms: Compositionality, Bimodal, Sparsity, Modality

\section{INTRODUCTION}

To be helpful teammates to human partners,

robots should be ready to robustly follow spoken directions. For example, an individual's supervisor may tell associate degree autonomous self-propelled vehicle, "Put the tire pallet on the truck," or the occupier of a chair equipped with a robotic arm may say, "Get me the book from the coffee table." Such commands are challenging because they involve events, objects and places, each of which must be grounded to aspects of the world and which can be composed in many alternative ways in which, we frame the matter of following directions as inferring the fore most seemingly mechanism state sequence from a language command.

Previous approaches assume that natural language commands have a fixed and flat structure that can be exploited when inferring actions for the robot. However, writing and maintaining ASCII text file could be a expensive business; computercode developers perpectually look into documenentation and on-line resources, and they need to make sense of large existing code bases. Both of those is difficult and bog down the event method.

Revised Version Manuscript Received on Jun 20, 2019.

Elakya.R, Department of CSE, SRM Institute of Science and Technology, Chennai, India.

Himanshu Sinha, Department of CSE, SRM Institute of Science and Technology, Chennai, India.

Shubham Gupta, Department of CSE, SRM Institute of Science and Technology, Chennai, India.

Singh Anubhav Gajendra, Department of CSE, SRM Institute of Science and Technology, Chennai, India.

Prince Kumar, Department of CSE, SRM Institute of Science and Technology, Chennai, India.
To make progress in this test setup, the robot would need to fulfill Jackendoff's Cognitive Constraint or possibly an automated understanding thereof. In particular, there should exist an exact outline that relates percepts to language and language to percepts because of generally the automated framework would nor be prepared to see its visual-etymological percepts nor execute its assignments. An imperative utilization of language process is that the translation of human headings.

The capacity to separate bearings and play out the implied activities is critical for wash cooperations with a workstation or a component. A portion of the ongoing work has investigated how to outline language guidelines into activities that can be performed by a PC.

\section{EXISTING SYSTEM}

Compositionality is enabled in this model across these projections and unobserved percepts are generalized. Let us assume a robot that manipulates little building blocks in a very work surface space. And then, this robot is tasked to human-vocal that navigates the development of non-trivial building-block structures.the robot must be able to interpret the; segment individual structures, spoken language (audio perception), orange rectangle, (visual perception) and then these may be able to identify and reason these collections of structures such that these should be relate such collections which can execute the action at right place.

\section{A. Disadvantages}

- This model enables distinct projections compositionally and So, on compositional basis it is spanned unobserved percepts.

- Assume a robot where small building blocks are manipulated in table top workspaces. Where this robot is let to be performed with pre-installed human commands such that it recognizes human vocals that guides the vocal structure and moves according to the command further.

- Audio Perception: This machine or the robot has to interpret the spoken language individual structures where the audio is structured and reasoned about the collection of structures.

- Physical modeling: And also it must be able to reason the physical structure like observing the physical shapes and properties of certain things and then the actions should be executed using these modeling. 


\section{PROPOSED SYSTEM}

The compositional nature of language to speak to bimodal visual-audial percepts portraying the scenes is misused in this framework. The bimodal portrayal is grounded in a language-based compositional model. Groupings of visual features are mapped to audio segments where we fix a two part structure. Hand tuning cannot be observed in specific mapping. All the elements of compositional model are jointly learned. This two-part integrative structure will show the shape of adjective-noun.

In this proposed system, the recognition is the main point where the complete system is integrated into different sensors through which the programs are loaded into the sensors for which the action is requested by the source of information.

Through this process, we come to know that the response the has to be given by the machine is more easier than the before existing system. This makes the proposed system to be more particular than the existing system. Here is why, the proposed system is consider as more faster when compared to that of existing system. The sensors used in the system can be of any type which depends according to the system that delivers the required output.

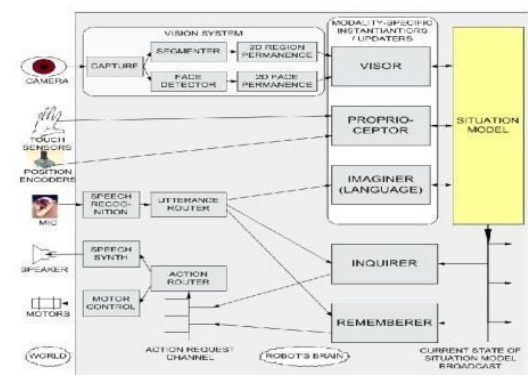

Fig 1. Proposed System

\section{A. Advantages Of Proposed System}

An earlier version, the entire system required a specific form of work to make the system consistent. Anyhow, now we look at the requirement and follow at generalized approach that suits this structure. This structure is hence fully induced by the data itself (what is inherent in the spoken language).

- Probabilistic Interpretation

- Sparse Representation

\section{SYSTEM ARCHITECTURE}

Our proposed system is designed in order to make the talk up between the Human or Source of Data and Robot easy. The data to the robot will be dumped into them by means of source of data like human through the sensors which are programmed to sense the data from the source.

The system architecture of the proposed system consists of different modules such as dictionary learning, Multi task learning and Pair sparse modeling. Apart from which the work flow in the system architecture which can be observed from the below diagram.

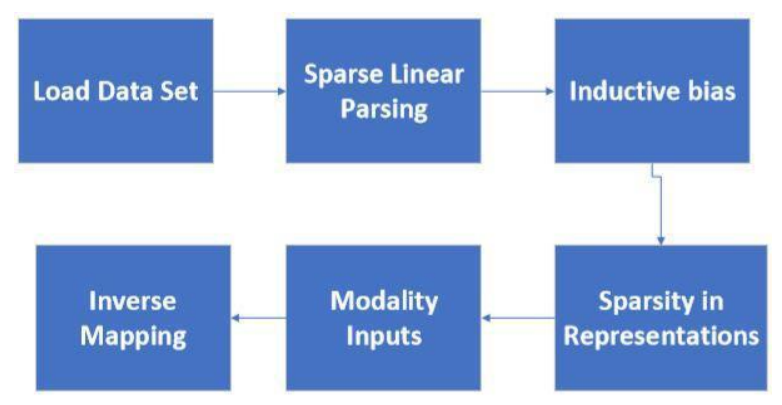

Fig 2. System Architecture (Work Flow)

The above mentioned is the work flow of the system architecture of the proposed system where the data set of instructions are loaded into the system. From then the loaded data set is parsed through sparse linear parsing from which inductive biasing is done. If the work flow till the this engaged then it is represented and Modular Inputs is given to them and finally inverse mapping is done to get the required output from the machine or the robot. This is the basic workflow of the proposed system to get the required output by passing through the mentioned procedures. Although the procedure to be followed is complex, the code given to the sensor should be perfect to recognize the data set that is to be loaded in to the system or device.

\section{SYSTEM MODULES}

\section{A. Dictionary Learning:}

This is method that is used to reconstruct a signal and also represents it, utilizing a meager direct blend of bases, which establish a word reference. The inadequate coding step is an underdetermined straight framework. As a result, sparsity on the remaking vector is utilized so as to acquire a computationally productive and authentic significant arrangement. Word reference Learning is the procedure whose objective is to locate a decent over-total premise as far as least guess blunder and sparsest arrangement given a lot of vector.

\section{B. Multi Task Learning:}

Inductive bias derived from other problem related to this makes the machine learning easier with the use of MTL. Multi-task learning has been used with success in most of the machine learning applications like natural language processing such as speech recognition to computer vision. MTL comes in many ways as follows: learning to learn, joint learning, and learning with auxiliary tasks are only some names are accustomed seek advice from it

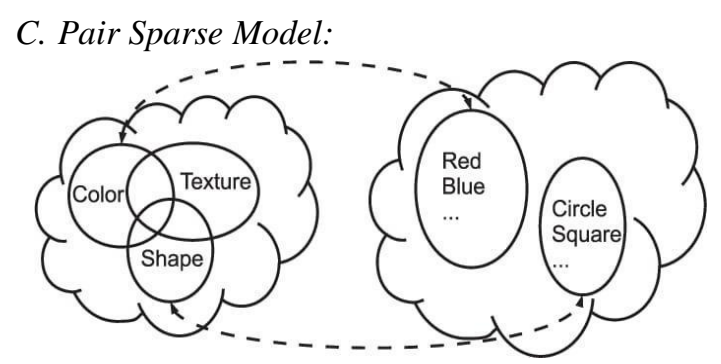

Fig 2. Pair Sparse Model 
Paired sparse modeling from neurobiology is driven into two findings as follows:

(i) Sparsity in representations

(ii) Modality inputs.

Because of which, we utilize matched lexicon getting the hang of, amid which individual tactile data is drawn or spoken to by an appropriated premise and thusly guaranteeing delineation shares coefficients over those bases. The accomplishment of combined word reference taking in envisioning pictures from highlights, picture super-goals, cross-style picture amalgamation, and past propelled us. Matched word reference learning is picked to our concern, for example, learning over-total lexicons for scanty bases in both the visual and sound area while utilizing similar coefficients crosswise over space bases. The online lexicon learning technique is utilized however the word reference refreshes and inadequate coding steps are elective strategies for that.

\section{PROBLEM STATEMENT}

There is increasing physiological proof that humans use sparse coding in illustration of various assorted sensory inputs. In this, sparse representation is used and interpretability is used as the signal is for minimizing a fit measure. And here, by the speculation of utilizing least vitality in neuron's excitation to speak to enter tangible information, sparse coding is used. Multi-modal sensory data is projected together on a common basis, similar as in compositional model, as evidences suggests the following data.

- Stimuli used to prepare the model comprised of instances of recorded discourse.

- The blue bend speaks to the crude sound weight waveform of a lady saying.

- The control range crosswise over acoustic frequencies is shown as a component of time, with hotter hues demonstrating high power substance and cooler hues showing low power.

- The spectrograms were then isolated into covering $216 \mathrm{~ms}$ portions.

- Subsequently, central parts investigation was utilized to extend each fragment onto the space of the initial two hundred chief segments.

The model represented below is used for the existing problem statement:

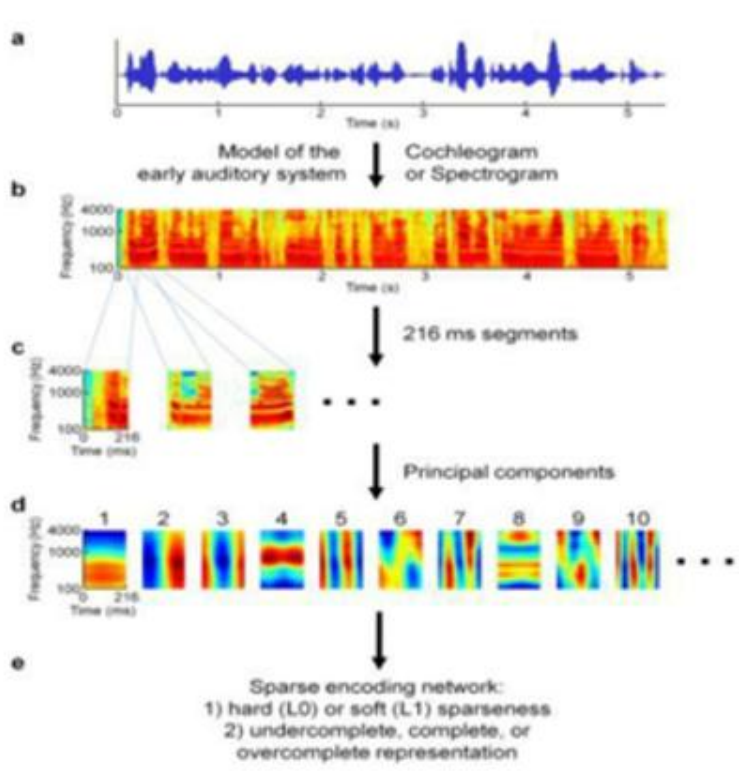

Fig 3. Sparse Encoding Network from Auditory system

\section{RESULT}

For proliferation, we look to assess the execution of a robot for a hypothetical order, e.g., pick a 'red square shape' from a crate brimming with every one of the shapes, which is a subset of the more extensive picture portrayed in the introduction. We play out a 3-overlap cross-approval concentrate to survey this recovery execution by separating the dataset into 3 sections, utilizing 2 sections for preparing and remaining part for testing (and afterward permuting the sets). We test recovery execution for various ideas (shading and shape) independently for combined meager learning and compositional inadequate learning. A shading or shape is resolved to be accurately comprehended by the robot. Recovery is performed by first separating the audial highlight from the audial stream, utilizing the educated word references and stage "pi" to remove visual highlights and after that picking the nearest object from all the preparation precedents.

\section{CONCLUSION}

From the above concepts, we come to an end and conclude the concept of our project as the robots which are programmed to work or respond to humans hear or visual the things that are projected on to the machine responds in such a way it picks the closest object from the programmed dictionary and from all the training examples. Here the performance of the robot depends on the programmed dictionary of paired sparse learning and compositional parse learning. There are also many future enhancements which are profitable with this kind of environment. This is what we conclude from our project work which makes artificial intelligenice and machine learning as the base of the proposed system. 


\section{ACKNOWLEDGMENT}

The authors would like to thank Mrs. Elakya.R for guiding throughout the project. The various ideas present here are through the work done at the development stages of this product.

\section{REFERENCES}

1. Learning to Interpret Natural Language Navigation Instructions from Observations : David L. Chen and Raymond J. Mooney ;Journal of Artificial Intelligence Research :2011.

2. A Joint Model of Language and Perception for Grounded Attribute Learning :Cynthia Matuszek, Nicholas FitzGerald, Luke Zettlemoyer, Liefeng Bo, Dieter Fox ; The Journal of Machine Learning Research:2012

\section{Authors Profile}

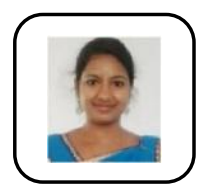

Elakya.R is currently a faculty advisor in SRM Institute of Science and Technology. Her current research interest is Computer Networks. She is currently in department of Computer Science and Engineering in Chennai, TN, India

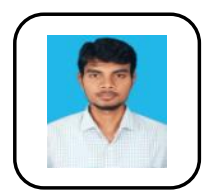

Himanshu Sinha is currently pursuing B. Tech (CSE) in his final year from SRM Institute of Science and Technology. He is currently working as an intern in Wipro Pvt. Ltd. in Chennai, TN, India

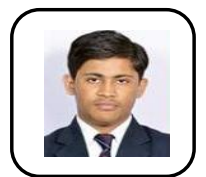

Shubham Gupta is currently pursuing B. Tech (CSE) in his final year from SRM Institute of Science and Technology. He is currently working as an intern as a job profile of front-end developer in Noida, UP, India

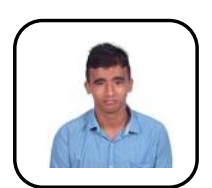

Singh Anubhav Gajendra is currently pursuing B. Tech (CSE) in his final year from SRM Institute of Science and Technology. He is currently working as an intern in Cognizant Technology Solutions in Chennai, TN, India.

Prince Kumar is currently pursuing B. Tech (CSE) in his final year from SRM Institute of Science and Technology. He is currently working as an intern in Infosys Ltd. in Mysore, KA, India. 